Article

\title{
Smart Cities in Taiwan: A Perspective on Big Data Applications
}

\author{
Shiann Ming Wu ${ }^{1}$ (D), Tsung-chun Chen ${ }^{1}$ (D), Yenchun Jim Wu ${ }^{1,2, *}$ (i) and Miltiadis Lytras ${ }^{3,4}$ \\ 1 College of Business Administration, National Huaqiao University, Quanzhou 362021, China; \\ wuming.hqu@gmail.com (S.M.W.); chentsungchun@hotmail.com (T.-c.C.) \\ 2 Graduate Institute of Global Business and Strategy, National Taiwan Normal University, \\ Taipei 10645, Taiwan \\ 3 Management Information Systems, The American College of Greece, Athens 15342, Greece; \\ mlytras@acg.edu \\ 4 Distinguished Scientist, King Abdulziz University, Jeddah 21589, Saudi Arabia \\ * Correspondence: wuyenchun@gmail.com; Tel.: +886-2-7734-3996
}

Received: 26 November 2017; Accepted: 2 January 2018; Published: 4 January 2018

\begin{abstract}
In this paper, we discuss the concept of a smart city based on information and communication technology (ICT), analyze the objectives of smart city development in Taiwan, and explain the supporting technologies that make such development possible. Subsequently, we propose a hierarchical structure framework of smart city systems with levels of complexity ranging from low to high and interconnections and interactive relationships in five dimensions: the Internet of Things (IoT), cloud computing, Big Data, Mobile Network, and smart business. We integrate each key resource of the core operation systems of cities to promote the innovative operation of cities and further optimize city development. We then propose a Big Data platform data flow framework that uses information from ubiquitous sensor networks and information equipment to analyze the Big Data application process of smart cities and determine the resulting advantages and challenges. Additionally, we analyze the current state of development of smart cities in Taiwan. Finally, we discuss a new philosophy of smart city development and provide a practical blueprint for the formation, operation, and development of the smart cities with the aim of creating a bright future for the smart cities of Taiwan.
\end{abstract}

Keywords: smart cities; big data; Internet of Things; mobile network; information and communication technology; Taiwan

\section{Introduction}

\subsection{The Smart City}

A city's various infrastructure investments are all oriented toward sustainable development, enhancing the quality of life of city residents, and increasing the city's competitiveness. As the world becomes increasingly datafied, cities are becoming digital replicas of the cyber worlds in which people reside [1], and the development and application of information and communication technology (ICT) is playing a decisive role in this transformation. The expansion of Big Data and development of the Internet of Things (IoT) are increasing the feasibility of smart city initiatives [2]. Cloud computing platforms store large amounts of data, handle computing, analysis, and decision-making processes, and perform automation control based on the results of these analyses and processes [3]. Smart cities are the realization of digital city development based on ICT infrastructure and employ Big Data, the IoT, and cloud computing. In the five-tier structure of a smart city's operation cycle, we collect data on IoT as the bottom-tier device of the smart city. After all data are collected and converted for cloud computing, the IoT can integrate the useful data into a big database for storage, analysis, and 
computation. Next, through a mobile network, the results will be presented in various forms, such as reports and webpages, in order to answer users' inquiries and fulfill the application needs of top smart businesses. This entire structure cycle will form the operational function of the smart city. Figure 1 is the five-layered framework of a smart city, which we will explain by different layers in the paper. A smart city is a structure with the core of big data, as is presented in Figure 1. This paper introduces its functions and concepts in the following sections.

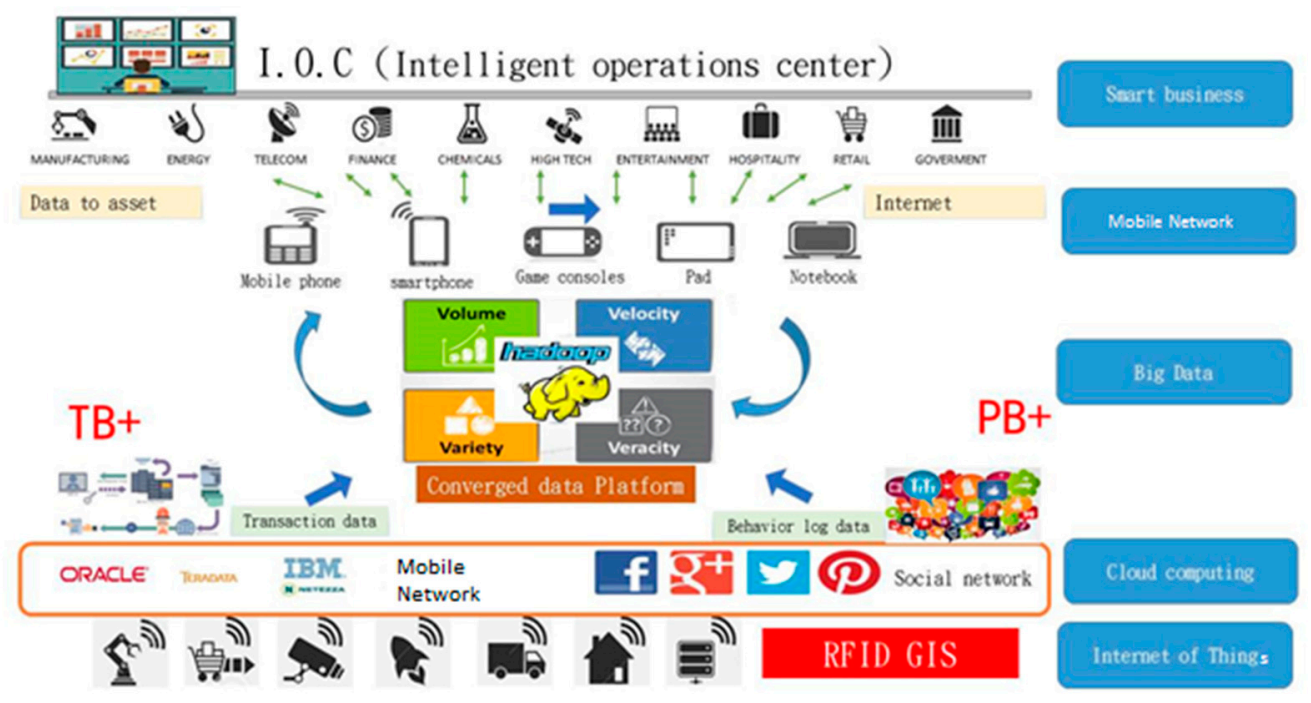

Figure 1. Enterprise application architecture of smart cities.

\subsection{Big Data}

The world is becoming increasingly datafied, and ubiquitous digital data on all aspects of human life have emerged in analyses of human activity rhythms and the digital breadcrumbs left by human activity. Data activation and the analysis of applications are essential in smart cities. Big Data is extensive datasets-primarily in terms of their volume, variety, velocity, and/or variabilitythat require a scalable architecture for their efficient storage, manipulation, and analysis [4]. Different concerns may be encountered during the capture, storage, management, mining, and analysis of Big Data depending on the usage context. Banks worldwide use Big Data to achieve their sustainable development goals. Big Data is also used for the following:

- to foster decision-making and accountability;

- to determine where funds are sent;

- to determine whether funding is being sent to the right place;

- for monitoring and evaluation;

- to identify changes over time;

- to determine whether such changes were caused by a specific intervention;

- to identify other factors that may have caused the outcome [5].

Big Data from satellite imagery and sensor networks are enabling increased measurement of environmental and developmental indicators [5]. There are 6 billion mobile phones in use worldwide, and $80 \%$ of them belong to people in developing countries. This prevalence provides researchers with a network of social sensors that are constantly obtaining information every day [6]. Big Data is a revolution that will change the ways in which people live, work, and think. Following Mobile Network and Cloud Computing, Big Data has gradually become a technology that has a far-reaching influence on the ICT industry [7]. 


\subsection{ICT}

The primary functions of ICT are terminals with data input and output capabilities, networks with data transmission capability, and computing power with data accumulation, processing, and analysis capabilities. In 1998, the Organization for Economic Cooperation and Development defined ICT as a combination of the manufacturing and service industries for electronic capture, transmission, data display, and electronic information. In 2016, the China Academy of Information and Communications Technology divided categories of the ICT industry into the types narrow and broad. Narrow industries included those of electronic components (integrated circuits, discrete devices, display devices, printed circuit boards, and other components), control devices and measurement instruments (equipment and instruments), terminal devices (mobile phones, personal computers or tablets, televisions, and wearables), cable devices (telecommunication network equipment), and cloud devices (servers, memory systems, and enterprise network equipment). Conversely, broad industries include those of software (basic software, application software, software especially used in vertical domains) and IT services (commercial IT services and IT product support services). By 2020 in the ICT industry, clouds (Big Data) and terminals (smart hardware) are predicted to become vertically stackable using the vertical integration (cloud and terminal) model of a horizontal platform [8]. Japan's ICT strategy focuses on IoT and artificial intelligence (AI) investments in Big Data and other telecommunication technologies to assist related firms achieve revolutionary innovation in the ICT field. The Industrial Economics and Knowledge Center (IEK) stated that the primary concept of Taiwan's ICT industry in 2017 is “The AI X Era Begins: Digital Innovation, AI Synergy". In a post-IoT era, AI smart devices are expected to gradually enter our lives, and the focus of industry will transfer from the IoT to AI [9].

\subsection{Planning of Smart Cities in Taiwan}

By applying innovative ICT, Taiwan continues to promote various programs related to smart technology and plans to build a modernized smart city that is low-carbon, green, and harmonious and has sustainable development. The IEK indicated that the development of smart cities must proceed from the perspective of solving problems to meet market demands and have the opportunity to create value and foster industry. Su et al. [10] expressed that after a global economic recession and the rise of local economy, the development of smart cities worldwide has gradually shifted to encouraging civic participation, using a bottom-up design, and employing an innovative economic model of digital economic value added (EVA). The benefits of smart city development can be viewed in four dimensions: ecological sustainability, social sustainability, economic sustainability, and governance [10]. The attitudes to the environment of citizens and firms actively participating in smart city development are of great importance [11]. The "Smart City Introduction Reference Manual" compiled by IDEAS (department of Institute for Information Industry, III) in 2012 details the eight primary application items of smart cities: smart education, smart tourism, smart transportation, a smart sustainable environment, smart health care, smart governance, smart and happy living, and smart industry. In 2015, the Executive Yuan of Taiwan began drafting the "ide@ Taiwan 2020 Policy: White Paper" and proposed five dimensions and 18 measures for implementation to forge a new Taiwan that is high-quality, innovative, and sustainable [12].

\section{Development of a Smart City}

Cities are densely populated areas with highly developed industry and commerce. They generally include functional zones such as residential, industrial, and commercial areas and have administrative jurisdiction. Smart cities use information technology in these zones to provide a variety of convenient smart applications and services for promoting more coordinated societal and natural development. 


\subsection{Development Indicators and Rating Mechanisms of International Smart Cities}

The concept of a smart city continues to spread around the world. Governments have successively created relevant policy guidelines and urban vision plans. Furthermore, because development is different for each city due to the natural environment and social background characteristics and because long-term efforts and resource investment are required, a variety of smart city indicators and rating mechanisms have been developed internationally to ensure that the strategies employed are economic and effective. This paper discusses only the four indicators and rating mechanisms commonly used by Taiwan's neighboring countries (Table 1). They are as follows:

- The European Smart City Index [13] employs the dimensions smart economy, smart people, smart governance, smart mobility, smart environment, and smart living and refines them into different indicators, assigning weights to assist ratings.

- After an overall assessment and formation of indicators, the Intelligent Community Forum (ICF) [14] promotes city or community management philosophies and operating models to city leaders. Additionally, they commend the achievements of cities in using ICT to develop a smart city through the ICF Awards, promoting exchange and learning between cities.

- $\quad$ SMART [15], the theoretical model of China's smart city evaluation indicator system, comprises five key elements: service, management and maintenance, application platform, resources, and technology.

- The Foreseeing Innovative New Digiservices (FIND) model [16] was designed by the Institute for Information Industry in hopes of employing rational research data and physical examination analysis to enhance the quality of life of urban residents and firm competitiveness.

Cities in Taiwan have obtained ICF smart city ratings on several occasions and have received different awards.

Table 1. Indicators and rating mechanisms commonly used by Taiwan and neighboring countries.

\begin{tabular}{|c|c|c|c|c|c|}
\hline Rating Name & First Use & Place of Origin & Originating Institution & Indicator Orientation & $\begin{array}{l}\text { Object of } \\
\text { Evaluation }\end{array}$ \\
\hline $\begin{array}{l}\text { European Smart } \\
\text { City Index }\end{array}$ & 2007 & $\begin{array}{l}\text { Austria/Slovenia/ } \\
\text { the Netherlands }\end{array}$ & $\begin{array}{c}\text { Vienna University of } \\
\text { Technology, University } \\
\text { of Ljubljana, Delft } \\
\text { University of Technology }\end{array}$ & $\begin{array}{l}\text { Smart economy, } \\
\text { smart people, } \\
\text { smart governance, } \\
\text { smart mobility, } \\
\text { smart environment, } \\
\text { smart living }\end{array}$ & $\begin{array}{l}\text { Medium-sized } \\
\text { cities in Europe }\end{array}$ \\
\hline SMART & 2013 & China & $\begin{array}{l}\text { China Software } \\
\text { Testing Center }\end{array}$ & $\begin{array}{c}\text { Intellectualized city } \\
\text { infrastructure, } \\
\text { intellectualized city } \\
\text { management, } \\
\text { intellectualized city services, } \\
\text { open information } \\
\text { resource integration, } \\
\text { intellectualized city industry }\end{array}$ & Cities in China \\
\hline FIND & 2010 & Taiwan & $\begin{array}{l}\text { Foreseeing Innovative } \\
\text { New Digiservices }\end{array}$ & $\begin{array}{l}\text { Intellectualized city ability, } \\
\text { citizen satisfaction, } \\
\text { benefits of intellectualized } \\
\text { city construction }\end{array}$ & Cities in Taiwan \\
\hline
\end{tabular}

Source: [13-16], organization of this study.

\subsection{Smart City Commercial Application Structure}

Following IoT development, Big Data is used as a core to compose the application architecture of smart cities. The applications of Big Data in various fields were determined in this study, and after summarization and organization, the enterprise application architecture of smart cities was constructed and is shown in Figure 1. 
- The underlying sensing equipment (e.g., Internet of Vehicles (IoV), QR codes, IP Cam, radio-frequency identification (RFID), eTag, and geographic information systems (GIS)) generate data, which are collected and converted using the interface.

- The IoT technology is used to collect information. Information is gathered through mobile networks or social networks into a database or data warehouse (e.g., Oracle, DB2, and Teradata) for information-related storage cloud operations.

- Subsequently, after extraction of various types of databases, transaction data and behavior log data are separately imported into the big database (such as Hadoop) of the converged data platform. This is the core of Big Data.

- After processing of the data, customers can use the Mobile Network to search for information.

- Various types of smart business applications are thus generated.

The convenience of the Mobile Network has contributed to the booming business applications in smart cities.

\subsection{Mobile Network}

The searchability of network attributes establishes the relationship between geographical proximity and social interaction [17]. City data are approximately divided into the categories of cartographic data, public data, and commercial data. These types of data are widely used and are provided by the Big Data platform and resource core in the information-based infrastructures of smart cities. The popularization of various new types of smart mobile devices has resulted in the generation of massive volumes of data. The rapid development of the Mobile Network has resulted in explosive growth in the number of smartphone users, number of apps, and the volume of network data traffic. According to statistics determined by the market research firm Sensor Tower regarding app downloads, the number of iOS and Android app downloads in 2016Q1 was 17.2 billion, and gaming and social interaction apps were the most downloaded app types [18].

Mobile Networks are gradually permeating people's lives and workplaces and has become a new channel for people's living, working, and entertainment, online or offline. The rapid development of Mobile Networks was the cornerstone of mobile Big Data. The sharp increase in the number of mobile apps has spurred the generation of massive user data, and ubiquitous access to information anytime has enabled Mobile Networks to become handier in daily life.

Services are available to users anytime and anywhere via the Internet, thanks to big data and the results of cloud computing.

\subsection{Cloud Computing}

Cloud computing is not a new technology or technique, but a concept [19]. The cloud represents the Internet. The Internet now provides substitutes for the software installed on computers and acts as an external hard disk space; the cloud in turn uses Internet services to perform various tasks and stores file data in a massive virtual space. According to the National Institute of Standards and Technology (NIST) sp800-145 definition, cloud computing is a model for enabling ubiquitous, convenient, on-demand network access to a shared pool of configurable computing resources (e.g., networks, servers, storage, apps, and services) that can be rapidly provisioned and released with minimal management effort or service provider interaction [20]. In October 2014, the International Organization for Standardization (ISO) published ISO/IEC 17788 and ISO/IEC 17789, which both propose a standard definition of cloud computing and frames of reference. Furthermore, the roles of cloud computing, cloud computing activities, and cloud computing components, as well as the relationships between them, were clearly described. Cloud computing is provided as a service to customers through the Internet.

In October 2015, the Executive Yuan launched a cloud computing development plan [21]. The government plans to use Taiwan's existing ICT industry advantage to consider Taiwan's industrial 
needs and the development of its application services. Trusted group procurement will be employed and the use of existing cloud computing solutions will be centralized to help industries establish economies of scale. Simultaneously, cloud computing application infrastructures will be established in related industries to accelerate the adoption of data development, Big Data, the IoT, smart cities, and Industry 4.0. Five strategies have been proposed: the promotion of public sensor application, expansion of application development power, establishment of software research and development technology, implementation of a cloud infrastructure, and consideration of the efficiency of green energy.

Information security has been predicted to be an obstacle to sustainable computing in the future [22]. The development of cloud computing has resulted in more convenience in the lives of ordinary people. However, information security and personal information privacy in cloud computing services must be carefully evaluated.

Big data and cloud computing enable IoT devices to have ultimate functions. Data produced by such devices can be stored in big data for ready application by a big data processing module after format conversion.

\subsection{The IoT}

According to IEEE P2413, the IoT has three layers: a sensing layer, networking and data communications layer, and applications layer. Accordingly, the International Telecommunication Union endorses the definition of the IoT as a network that is "available anywhere, anytime, by anything and anyone". In this context, consumer products can be tracked using minute radio transmitters or tagged or embedded hyperlinks and sensors [23].

The interconnection of the IoT with smart devices, the upgrades of cloud technology, an easy-to-operate natural human-machine interface, and a good user experience can be combined with various types of media content such as broadcast television, video, audio, text, images, and data. Various crucial applications can be integrated to provide multimedia services with high service quality, a favorable user experience, and high security, interactivity, and reliability; these are the factors that increase customers' willingness to make a purchase. Available technology and system architectures are used to effectively integrate the relevant signals, networks, and protocols of the underlying sensors and micro-components. Then, using the operating system, the middleware, cloud solutions, application interfacing, information management, and the Big Data of the software architecture, most devices should have self-management and self-optimization capabilities. In this manner, the need for automatic transmission of data in the complex IoT system can be met. The IoT acts as a bridge connecting people with machines and machines with machines [24].

The IoT is one of the largest sources of Big Data, and when large amounts of data require high-frequency processing, transmission, and analysis, the IoT and Big Data interact to transmit and store data. "Data security and privacy will play an important role in IoT deployments. Because IoT systems will produce and deal with personally identifiable information, data security and privacy will be critical from the very beginning [25]".

The IoT is the creator of data, and different types of data can be useful information after format conversion. Making IoT smart renders the data it produces more meaningful. Therefore, big data can be available. We offer a more detailed introduction to big data in Section 3.

\subsection{Range of Application of Smart Cities in Taiwan}

Following Cohen [26], we measure the development outcomes of a smart city from six dimensions. We hereby summarize the opinions of experts and scholars in all cities of Taiwan and the application and service programs are summarized as follows. Smart government includes weather forecast system, population census, open data platform, integrated disaster prevention system and waste management, electronic voting, smart city operation center, and smart policing. Smart environment consists of energy demand response system, solar photoelectricity, wind power generation, smart meter, exploration, collection and storage, smart water resources, water pollution monitoring, earthquake 
early warning system, forest fire prevention, and air quality monitoring. Smart living is composed of the digital convergence of video and audio platforms, accessibility services, smart tourism, mobile citizen cards, e-health information, smart hospitals, medical applications of artificial intelligence, care robots, and smart kits. Smart mobility includes in-car Internet, smart transport system ETC applications, smart parking lots, traffic flow, traffic detection, logistics vehicles and cargo management, smart retail, cross-border custom clearance, agricultural product traceability, and smart packaging. Smart people consist of smart learning, smart administration, smart health care, smart community, smart energy, smart home care, home safety control, smart community, and smart care. Smart economy is composed of smart finance (payment, insurance, financing, fundraising, investment management, and market supply). Cities shall prioritize these applications based on their respective characteristics and budget and work with available enterprises to implement such applications.

\section{Big Data}

The operation of big data is the core of smart city construction. First, data collection and awareness should be enhanced [1]. Cities should improve their data awareness and promote the extensive application of new technologies, such as wireless identification technologies, sensors, and wireless networks, to achieve smart infrastructure and smart city operation and management. Second, digital resources should be further integrated and different government departments should share data with each other [12]. Third, attention should be paid to the training of professionals in cities, and industrial advantages should be fully tapped. The construction of smart cities will certainly contribute to progress in big data [3]. In the future, the application market of big data in smart cities will constantly grow, and external development and exchange should be expanded. Concentration and development of the urban industrial chain should be fueled with the promotion and application of innovative technologies. Finally, we should also attach importance to setting up a sound data security system and strengthening network security protection. Data assetization is an inevitable trend, and data about the internal operations of enterprises and customer data are valuable data assets. Therefore, it is necessary to improve the security of urban information network. At the same time, we should also establish security monitoring and emergency response mechanisms [22], coordinate departments for monitoring and prevention, and thwart and reduce network threats to public and enterprise networks.

\subsection{Big Data Management}

Data can generally be divided into structured data, semistructured data, and unstructured data. Preprocessing unstructured data, converting them into structured data, and then storing the data is highly crucial for subsequent data mining. Complete use of Big Data depends on effective management to facilitate subsequent mining and cross analysis. A distributed file system (DFS) is a type of file system that allows files to be shared across multiple hosts using a network and enables multiple users on multiple machines to share files and storage space. The industry often uses the NIST SP800-1500 Big Data specifications as a template for operation management. The Hadoop DFS (HDFS) is widely used in the industry. Employing different designs, Big Data can be efficiently stored, managed, mined, and analyzed.

Building a data-centric smart city requires the vigorous support of data mining technology and the integration of different data formats. The application of data is the key to conducting data mining research on smart cities. Data visualization technology can present complex city data to customers in a concise and orderly form, favorably filling the understanding gap between the technology and its users.

\subsection{Big Data Activation and Application}

The data mining of business intelligence use statistics, AI, or other analytical techniques in the depths of Big Data is employed to identify unknown or hidden relationships and regulations. This achieves classification, estimation, prediction, affinity grouping, and homogeneous grouping, 
followed by presentation to the user using methods such as reports or web portals. This paper organizes the framework of Big Data applications in Taiwan's general industries as illustrated in Figure 2.

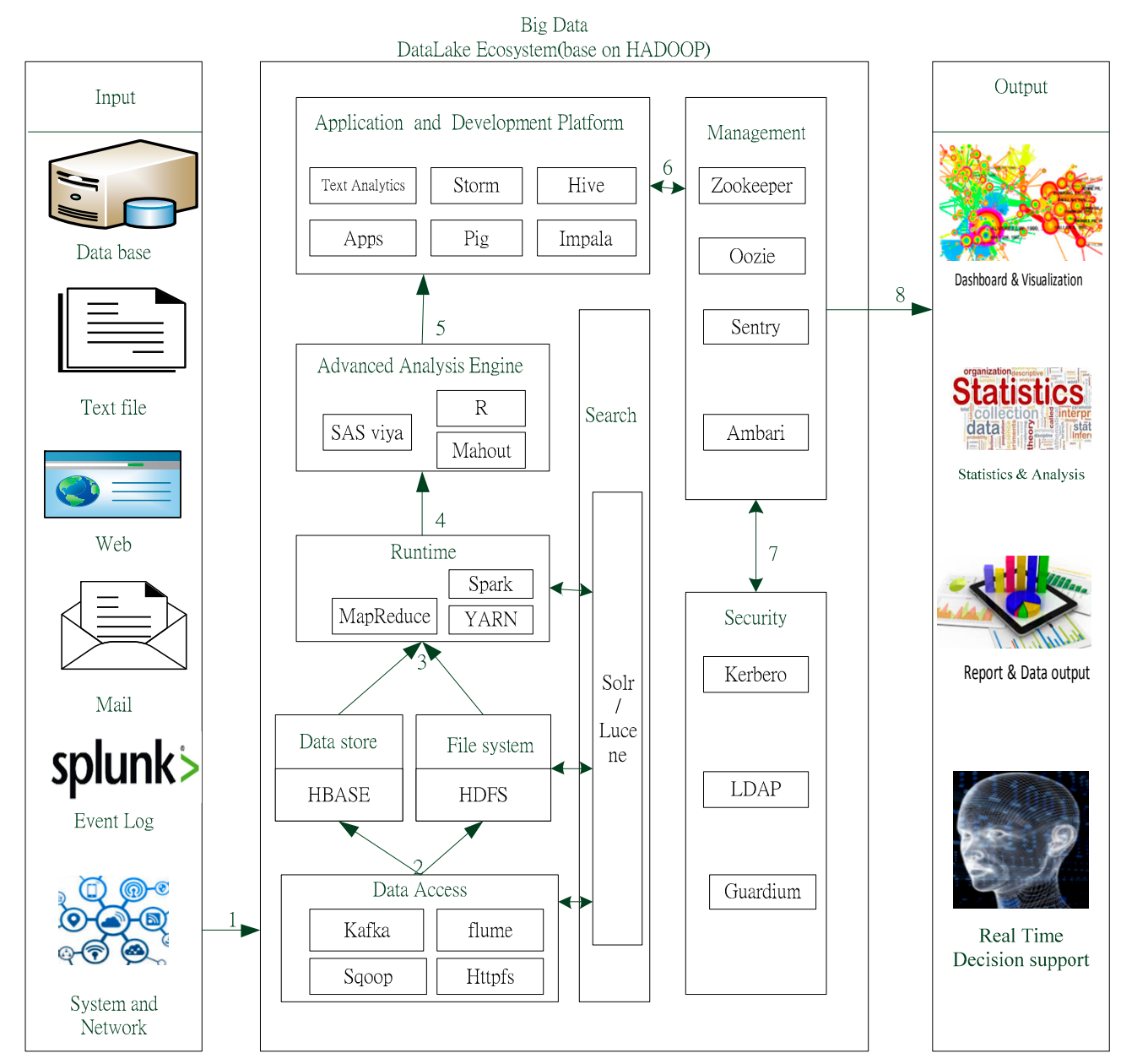

Figure 2. Big Data operations model.

The Big Data operational process, data flow sequence, and various functions of the platform in Figure 2 are described as follows:

- Input: Different types of data are input through the system and network interface to the data access module.

- Data Access: For the access interface, which is responsible for connecting to external heterogeneous platforms and applications, the commonly used kits and functions are Kafka distributed messaging, Sqoop for relational database connectivity, flume data clustering, and HttpFS (Hadoop Distributed File System, HDFS) supported HTTP (Hypertext Transfer Protocol) links and operations.

- The data store is the primary data storage system for a Big Data platform. HBASE is the database system used by Hadoop. The file system is the primary file storage system for the Big Data platform, and HDFS is the DFS (Distributed File System) used by Hadoop.

- RUN TIME is primarily responsible for parallel operations and program execution. MapReduce processes large volumes of data for Hadoop and complies with system architecture with a high fault tolerance. Spark is a cluster computing framework that uses in-memory computing technology. YARN (Hadoop MapReduceV2) processes large volumes of data for Hadoop 2.0, complies with system architecture with a high fault tolerance, and is also called MapReduce 2.0. 
- The advanced analysis engine is used for kits related to statistical analysis and machine learning. $\mathrm{R}$ language is employed for statistical analysis and machine learning. Mahout and SAS Viya are approximately similar; it is most convenient to use the MapReduce function library for distributed analysis and machine learning operations.

- The application and development platform uses related development tools for various developments and applications of Big Data. Text Analytics is employed for text mining and analysis, and Apps is used for mobile application development. Storms is a fault-tolerant distributed open-source computing system and Hive uses SQL syntax to access Hadoop data; Pig is employed for data manipulation and processing, and Impala is used for high-performance SQL-like access of Hadoop data.

- Management: The collaborations between each component and resource management include the monitoring and setting of files, databases, and system resources. ZooKeeper collaborates with the internal servers of Hadoop, and Oozie is a workflow manager on Hadoop. The purpose of Sentry for Apache Sentry is to handle privilege management; it is a policy engine used by data processing tools to verify access rights. Ambari is a tool for establishing, managing, and monitoring Hadoop clusters.

- Security: The system security is responsible for authorization, verification, and encryption tools. Kerberos is a network authentication protocol used for secure identity authentication of personal communication on insecure networks. LDAP is an open, neutral, and industry-standard application protocol that uses IP protocol to control access and maintain the directory information of distributed messages. Guardium automates all compliance workflows in a heterogeneous environment to ensure the privacy and integrity of reliable information in the data center.

- The output module renders outputs using visualization, statistical graphs, and reports.

The aforementioned steps form the data flow cycle of the Big Data platform. This cycle must be supported by infrastructure such as the IoT, cloud computing, and a Mobile Network, synergizing commercial applications.

\section{Current Developments in the Smart Cities of Taiwan}

A secondary qualitative study was employed in this paper for research and analysis, and we needed to garner tons of reliable data. Therefore, we visited official websites of governments at all levels via the Internet and downloaded public budget statements, plans, policy plans, and other electronic documents of official public data and summarized budgets, infrastructure expenses, and enterprise investments of different cities. We analyzed and integrated these data in different years to probe into the strengths and weaknesses of smart city projects in six major cities of Taiwan.

This study uses the ICF's five dimensions to evaluate six special municipalities in Taiwan (New Taipei city, Taipei city, Taoyuan city, Tainan city, and Kaohsiung city) and promote research on the developmental characteristics of smart cities (due to the small size of cities in Taiwan, collaboration, leadership, and sustainability are largely similar and are not included in the statistical evaluations). The demographics of the cities are detailed in Table 2, and the developmental characteristics of the cities are shown in Table 3.

Table 2. Demographics.

\begin{tabular}{cccccc}
\hline City & Area $\mathbf{( k m}^{\mathbf{2}} \mathbf{)}$ & Population & $\begin{array}{c}\text { Population Density } \\
\left(\text { Person/ } \mathbf{k m}^{\mathbf{2}}\right)\end{array}$ & $\begin{array}{c}\text { Number of } \\
\text { Motor Vehicles }\end{array}$ & Number of Factories \\
\hline New Taipei city & 2053 & $3,970,644$ & 1934.48 & $3,193,363$ & 18,251 \\
Taipei city & 272 & $2,704,810$ & 9951.48 & $1,756,877$ & 1098 \\
Taoyuan city & 1221 & $2,105,780$ & 1724.70 & $1,877,439$ & 10,297 \\
Taichung city & 2215 & $2,744,445$ & 1239.08 & $2,725,634$ & 16,955 \\
Tainan city & 2192 & $1,885,541$ & 860.33 & $1,947,077$ & 8621 \\
Kaohsiung city & 2952 & $2,778,918$ & 940.50 & $2,879,176$ & 6992 \\
\hline
\end{tabular}

Source: Yearbook 2016, [26-30]. 
Table 3. Developmental characteristics of the six cities.

\begin{tabular}{|c|c|c|c|}
\hline City & $\begin{array}{l}\text { Developmental } \\
\text { Characteristics }\end{array}$ & Implementation Characteristics & Current Status \\
\hline New Taipei city & Mobile applications & $\begin{array}{l}\text { Perform public service mobilization and informatization, } \\
\text { reduce the urban-rural information gap, and increase } \\
\text { public service efficiency and the degree of } \\
\text { public participation }\end{array}$ & Implementation stage \\
\hline Taipei city & $\begin{array}{l}\text { Network convenience } \\
\text { Mobile applications }\end{array}$ & $\begin{array}{l}\text { Promote the Taipei free (TPE-Free) wireless Internet in } \\
\text { the city and use the open data platform to create an } \\
\text { innovative industry development environment }\end{array}$ & Implementation stage \\
\hline Taoyuan city & Regional development & $\begin{array}{c}\text { Use the Taoyuan Aerotropolis as a center, promote } \\
\text { various smart applications, and diffuse and copy } \\
\text { successful models to corners of the city }\end{array}$ & Planning stage \\
\hline Taichung city & Industry investment & $\begin{array}{l}\text { Use vigorous local commercial power; the introduction } \\
\text { of crucial large-scale construction and investment in key } \\
\text { economic parks will create innovative industrial } \\
\text { development environments and create a new look } \\
\text { for the city }\end{array}$ & $\begin{array}{c}\text { Planning and } \\
\text { implementation stages }\end{array}$ \\
\hline Kaohsiung city & $\begin{array}{l}\text { Humanistic } \\
\text { transportation }\end{array}$ & $\begin{array}{l}\text { Promote green transportation information services, } \\
\text { enhance public service efficiency, create a new mobile } \\
\text { objective for the city, and establish a favorable } \\
\text { environment that is low-carbon and sustainable }\end{array}$ & Implementation stage \\
\hline
\end{tabular}

Source: Chieh (2015) [31,32], organized by this study.

According to information contained in the 2017 Statistical Yearbook of relevant departments of the Executive Yuan and various city governments, a comparison of data from 2014-2016, after weighted conversion is presented in Table 4 (10 the highest, 0 the lowest). The crucial data items include:

- Broadband connectivity: Including subscribers of various telecom services and penetration rates of various telecom services (total 4G subscribers for mobile subscribers in 2014; mobile broadband subscribers including data transmission services for 3G, 4G subscribers and Wireless Broadband Alliance (WBA) subscribers; fixed networks including ADSL (Asymmetric Digital Subscriber Loop), FTTx (Fiber To The x), Cable Modem, leased lines and Public WLAN (PWLAN) subscribers; other broadband subscribers include PWLANs and leased lines) [29,30,33].

- Knowledge workforce: Calculated by the ratio of the following factors-Population by Educational Attainment, Education budget, Number of teachers, Number of college, Number of college students and graduate students, Ratio of Classes, Teachers and Students of All School Levels [34-40].

- Innovation: Calculated by the ratio of the following factors-Number and Capital of Companies Registered-by Organization and Industrial Classification, Number and Capital of Registered Business Enterprises, Annual Expenditure for General Budget and Financial Statement [27,28,34-39].

- Digital inclusion: Calculated by the ratio of the following Items-Cultural Activities Social Educations, the Special Education Taiwan Cultural and Creative Industry Classifications, Number of Cultural and Creative Industries [29,34-39,41,42].

- Marketing and advocacy: Calculated by the ratio of the following Items-Internet Services, the Principal Indicators of Family Income and Expenditures, Result of Promotion for Community Development/Revenue and Expense for Operations Funds, Tourist Businesses and Inbound Visitors, Number of Visitors to the Principal Scenic Spots, Number and Capital of Companies Registered-by Organization and Industrial Classifications, Actual Number of Personnel in the Organic Structure with Subsidiaries [27,28,34-39].

- Sum: The sum of above. 
Table 4. Statistical analysis scores of the five Intelligent Community Forum (ICF) dimensions for the six cities in Taiwan.

\begin{tabular}{ccccccc}
\hline & $\begin{array}{c}\text { Broadband } \\
\text { Connectivity }\end{array}$ & $\begin{array}{c}\text { Knowledge } \\
\text { Workforce }\end{array}$ & Innovation & $\begin{array}{c}\text { Digital } \\
\text { Inclusion }\end{array}$ & $\begin{array}{c}\text { Marketing } \\
\text { \& Advocacy }\end{array}$ & Sum \\
\hline New Taipei city & 9.12 & 5.8 & 6.8 & 0.2 & 7.0 & 28.92 \\
Taipei city & 9.46 & 9.2 & 2.7 & 6.2 & 6.3 & 33.86 \\
Taoyuan city & 9.13 & 4.5 & 1.3 & 3.9 & 2.9 & 21.73 \\
Taichung city & 9.14 & 6.3 & 1.4 & 8.2 & 5.5 & 30.54 \\
Tainan city & 8.8 & 5.8 & 1.2 & 9.8 & 5.7 & 31.30 \\
Kaohsiung city & 8.85 & 6.1 & 8.4 & 2.7 & 6.9 & 32.95 \\
\hline
\end{tabular}

Sources: 1. 2016 report of a survey on broadband Internet usage in Taiwan, TWNIC. 2. Directorate-General of Budget, Accounting and Statistics, Executive Yuan, Yearbook 2017; Yearbook of Environmental Protection Statistics, 2017 [30-32]. 3. 2017 Yearbooks-Ministry of Education (MOE), Ministry of Economic Affairs (MOEA), Ministry of Interior (MOI), Ministry of Transportation and Communications (MOTC), Ministry of Culture(MOC), Ministry of Education (MOE), National Communications Commission (NCC), New Taipei city, Taipei city, Taoyuan city, Taichung city, Tainan city, Kaohsiung city [33-39,41,42].

Explanation: The strengths of the five ICF dimensions are scored, with 0 points indicating the weakest and 10 points indicating the strongest in Figure 3.

Figure 3 illustrates the strengths of the six cities in the five dimensions. Regarding broadband connectivity, Taipei city was the most favorable, whereas Tainan city was the weakest; regarding knowledge workforce, Taipei city was again the most favorable, whereas Taoyuan city was the weakest; Kaohsiung city was discovered to have the most favorable innovation and Tainan city the weakest. For digital inclusion, however, Tainan city was the most favorable; New Taipei city was the weakest. New Taipei city had the most favorable marketing and advocacy, and Taoyuan city the weakest. The smart city implementation characteristics for which each city has made plans (as shown in Figure 3) can also be used to strengthen the cities according to these weaknesses.

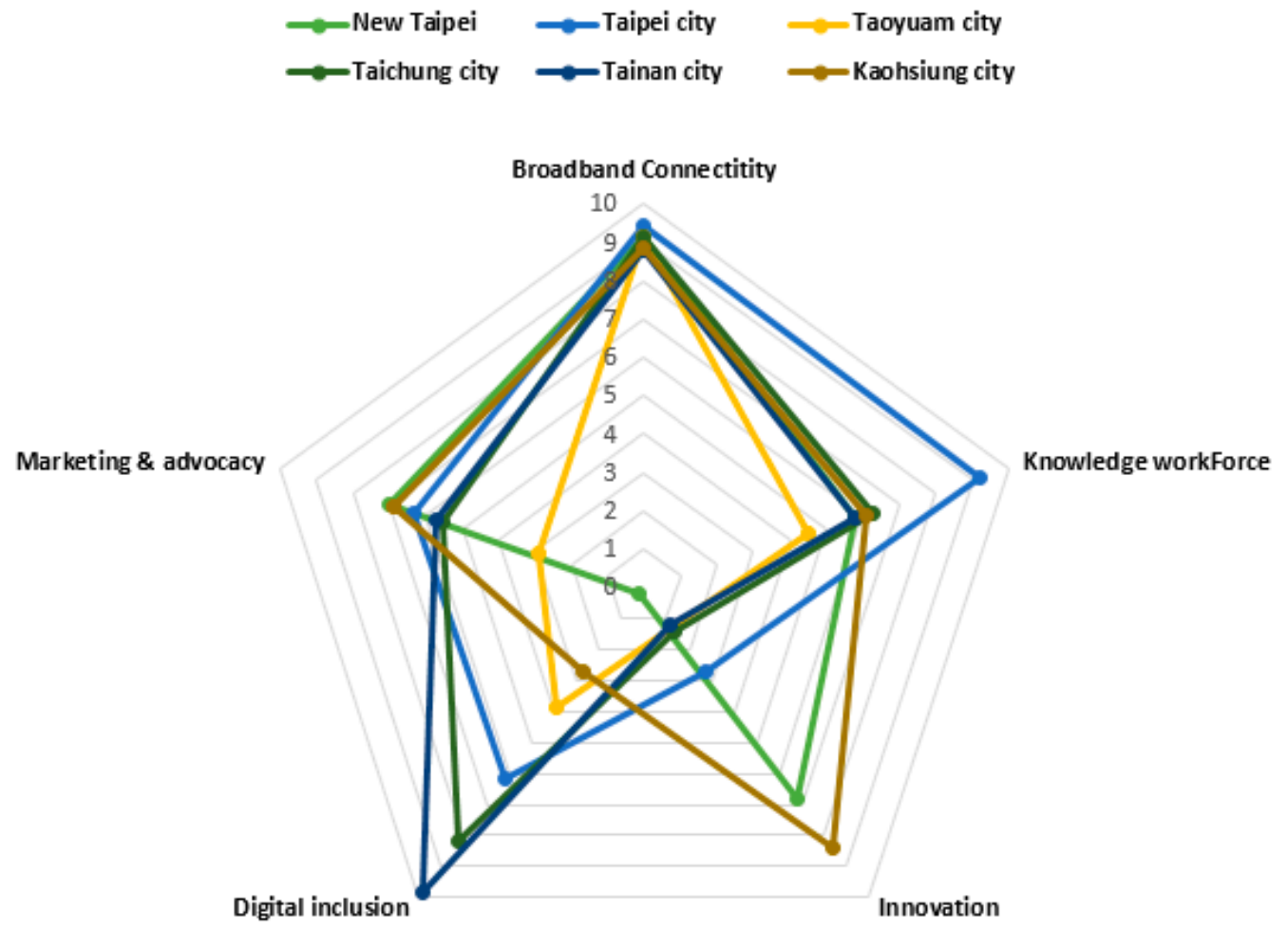

Figure 3. Strengths of the six special municipalities in the five dimensions. 
From the weighted scores of the statistical annual report analyses of each city, it was determined that Taipei city had the overall highest score (33.86 points), followed by Kaohsiung city (32.95 points), Tainan city (31.30 points), Taichung city (30.54 points), New Taipei city (28.92 points), and Taoyuan city (21.73 points). These results are in line with Taiwan's political and economic development profile. Taipei city was the first special municipality of Taiwan and is also Taiwan's political and economic center. Its overall development began the earliest and is mostly complete; in broadband connectivity and knowledge workforce, it ranks first of the six cities. Kaohsiung city was Taiwan's second special municipality and is the political and economic center of southern Taiwan. Its overall development is more complete than that of other cities, excluding Taipei city. Tainan city and Taichung city are provincial cities and cultural centers with relatively more cultural atmosphere; thus, their digital inclusion scores rank highly. New Taipei city and Taoyuan city are relatively new special municipalities; a larger gap between urban and rural residents and services exists within its area of jurisdiction. Because early government budgets (2010-2016) were relatively small and infrastructure development occurred relatively late, it is anticipated that these cities will catch up rapidly and increase infrastructure funds to balance regional development.

\section{Conclusions}

Whether the sustainable development of a city is judged successful is primarily based on residents' perception of space and their space requirements [43]. Smart cities are now being developed worldwide; the purpose of establishing various evaluation indicators is to uncover the individual characteristics and service capabilities of each city. Verifying the effectiveness of a smart city plan provides a direction that guides the development of other cities. Measurements of smart cities do not have a definite standard; if a smart city with favorable development performance in the past did not take advantage of ICT development and continue to uncover new horizons and produce innovative services, the function of the smart city may be relatively deceased.

Because each city in Taiwan is promoting intellectualization, it is necessary-in addition to referencing smart city evaluation indicators-to evaluate the local advantages and needs of Taiwan. Consolidating existing resources, utilizing local characteristics, planning the sustainable operation of cities, and increasing the convenience of residents' lives can produce a smart city that truly meets the needs of residents and ultimately results in a more favorable quality of life and friendlier living environment with sustainable development.

Due to the special island environment of Taiwan and its limited local resources, smart city development must be people-oriented and ICT advantages should be used to solve local issues. This study builds a reliable and general automated evaluation model [41] as a response to the question of how to use sustainable city planning for the effective use and distribution of resources. Second, $4 \mathrm{P}$ public-private partnerships should be formed to promote innovative entrepreneurship platforms and enhance economic growth. Third, interdepartmental management of the government must be conducted to promote mutual cooperation between each competency department and realize open data and the effective integration and maintenance of a Big Data platform. Fourth, planning for information security has been implemented. Big Data analysis equipment, environment, resources, and data collection followed by re-analysis and application must be ensured. During the process, due to the crucial confidentiality of customer information and how to de-identify personal information and the sharing of comprehensive precautionary measures, it is necessary to pay attention to whether data sources comply with confidentiality principles, whether the analysis method employed is suitable, and whether the Big Data analysis results are fully used to ensure the confidentiality of data and the rights of customers. Finally, under the influence of the new trend of smart city development, how to adjust traditional laws, regulations, rules, norms, and implementation standards in response to changes in planning methods is dependent on the efforts of government departments and large-scale enterprises in taking the lead for planning and implementation. The innovative smart city solutions resulting from digital technologies, such as smart energy, smart food security, and smart environments, must be applied properly to achieve the sustainable goal of smart cities with low-carbon, ecofriendly, green-energy, recycling, and livable environments. 
Acknowledgments: This manuscript was partially supported by Ministry of Science \& Technology, Taiwan (MOST 106-2511-S-003-029-MY3).

Author Contributions: Shiann Ming Wu designed the research and architecture, established the index system, completed the data statistics and analysis; Tsung-chun Chen provided some case research papers' references; Yenchun Jim Wu and Miltiadis Lytras contributed valuable assistance during the manuscript writing; Yenchun Jim $\mathrm{Wu}$ also assisted in the writing and modifying the manuscript formats. All authors read and approved the final manuscript.

Conflicts of Interest: The authors declare no conflict of interest.

\section{References}

1. Li, D.R.; Yao, Y.; Shao, Z.F. From digital Earth to smart Earth. Chin. Sci. Bull. 2014, 59, 722-733. [CrossRef]

2. Hashem, I.A.T.; Chang, V.; Anuar, N.B.; Adewole, K.; Yaqoob, I.; Gani, A.; Ahmed, E.; Chiroma, H. The role of big data in smart city. Int. J. Inf. Manag. 2016, 36, 748-758. [CrossRef]

3. Li, D.R.; Shan, J.; Shao, Z.F. Geomatics for smart cities-concept, key techniques, and applications. Geo-Spat. Inf. Sci. 2013, 16, 13-24. [CrossRef]

4. NIST Special Publication 1500-1. NIST Big Data Interoperability Framework: Definitions; NIST: Gaithersburg, MD, USA, 2015; Volume 1.

5. Big Data for Social Good LUCA Data-Driven Decisions. Available online: https://www.luca-d3.com/bigdata-social-good/index.html (accessed on 10 October 2017).

6. Anupam, A. The Big Data Revolution for Sustainable Development. In Proceedings of the XI Meeting of the Latin America and the Caribbean Monitoring and Evaluation Network, Santiago, Chile, 28-30 June 2016; Independent Evaluation Office, Global environment Facity: Santiago, Chile, 2016.

7. China Electronics Standardization Institute. Big Data Standardization White Paper; China Electronics Standardization Institute: Beijing, China, 2016.

8. China Academy of Information and Communications Technology (CAICT). ICT Manufacturing Development Report 2016. Available online: http:/ / www.caict.ac.cn/kxyj/caictgd/xxh/ (accessed on 22 October 2017).

9. Industrial Economics and Knowledge Center (IEK). Available online: http:/ /ieknet.iek.org.tw /ieknews / news_more.aspx?nsl_id=14f5326242074c95a3f07a6c514a0081 (accessed on 15 October 2017).

10. Su, S.; Chi, C.; Su, M.Y.; Chao, T.Y. Opportunities and Challenges with Digital Economy Trends. 2017. Available online: https:/ / www.itri.org.tw/Chi/Content/NewsLetter/contents.aspx?SiteID=1\&MmmID= 5000\&MSID=744474650526526402 (accessed on 20 October 2017).

11. Yu, J.; Lee, S. The Impact of Greenhouse Gas Emissions on Corporate Social Responsibility in Korea. Sustainability 2017, 9, 1135. [CrossRef]

12. National Development Council. Ide @ Taiwan 2020 (Creative Taiwan) Policy White Paper; National Development Council, Public Governance Quarterly: Taipei, Taiwan, 2015; Volume 3, pp. 93-100.

13. Europeansmart. Available online: http://www.smart-cities.eu/ (accessed on 18 October 2017).

14. Intelligent Community Forum. Available online: http://www.intelligentcommunity.org/ (accessed on 22 October 2017).

15. Smart city China. Available online: http:/ / www.smartcitychina.cn/ (accessed on 22 October 2017).

16. Foreseeing Innovative New Digi Services. Available online: https://www.find.org.tw/ (accessed on 20 October 2017).

17. Nicola, I.; Duccio, S.; Enrico, C. Smart Cities, Big Data, and Communities: Reasoning from the Viewpoint of Attractors. IEEE Access 2016, 4, 41-47.

18. 2016 Q1 Global APP Downloads TOP20 List. 21 April 2016. Available online: http://3c.yipee.cc/69866/ 2016-q1-\%E5\%85\%A8\%E7\%90\%83-app-\%E4\%B8\%8B\%E8\%BC\%89\%E9\%87\%8F-top-20-\%E6\%8E\%92\% E8\%A1\%8C\%E6\%A6\%9C\%EF\%BC\%8C\%E4\%BD\%A0\%E6\%9C\%89\%E5\%93\%AA\%E5\%B9\%BE\%E5\%80\% $8 \mathrm{~B} \% \mathrm{EF} \% \mathrm{BC} \% 9 \mathrm{~F}$ (accessed on 17 October 2017).

19. Huang, T.S. Talking about Cloud Computing. NTU ePaper, 20 March 2009; Volume 0008. Available online: http:/ / www.cc.ntu.edu.tw/chinese/epaper/0008/20090320_8008.htm (accessed on 12 October 2017).

20. Mell, P.; Grance, T. The NIST Definition of Cloud Computing, sp800-145, September 2011. Available online: https: / / www.tasclinx.com/wp-content/uploads/2013/02/141103-US-SP800-145.pdf (accessed on 12 October 2017). 
21. 2016-2018 Cloud Computing Development Programs. Board of Science and Technology, Executive Yuan, 5 October 2017. Available online: https:/ / www.bost.ey.gov.tw/cp.aspx?n=B56ED9F993B2EFA5 (accessed on 16 October 2017).

22. Choi, M. Leadership of Information Security Manager on the Effectiveness of Information Systems Security for Secure Sustainable Computing. Sustainability 2016, 8, 638. [CrossRef]

23. Abiy, B.C.; Domenico, R.; Roberto, M. Towards a Definition of the Internet of Things (IoT). 2015. Available online: https:/ / www.researchgate.net/publication/317588072_Towards_a_definition_of_the_Internet_of_ Things_IoT (accessed on 12 October 2017).

24. International Telecommunication Union. The Internet of Things; ITU: Geneva, Switzerland, 2005.

25. European Research Cluster on Internet of Things (TERC). IoT Business Models Framework. Deliverable 02.01. Available online: http:/ / www.internet-of-things-research.eu/pdf/D02_01_WP02_UNIFY-IoT_Final.pdf (accessed on 10 October 2017).

26. Cohen, B. Methodology for 2014 Smart Cities Benchmarking. Available online: https: / /www.fastcompany. com/3038818/the-smartest-cities-in-the-world-2015-methodology (accessed on 12 December 2017).

27. MOEA 2016 Yearbook. Available online: http://dmz9.moea.gov.tw/gmweb/investigate/InvestigateG.aspx (accessed on 20 October 2017).

28. MOI 2017 Yearbook. Available online: http://dmz9.moea.gov.tw/gmweb/investigate/InvestigateG.aspx (accessed on 20 October 2017).

29. NCC 2017 Yearbook. Available online: https://www.ncc.gov.tw/chinese/gradation.aspx?site_content_sn= 1967 (accessed on 12 October 2017).

30. A Survey on Broadband Internet Usage in Taiwan-A Summary Report. Taiwan Network Information Center, July 2016. Available online: https:/ / www.twnic.net.tw/download/200307/20160922e.pdf (accessed on 20 October 2017).

31. MOTC 2017 Yearbook. Available online: https://www.motc.gov.tw/ch/home.jsp?id=59\&parentpath=0,6 (accessed on 16 October 2017).

32. Executive Yuan 2017 Yearbook. Available online: https://www.dgbas.gov.tw/lp.asp?CtNode=3120\& CtUnit=1049\&BaseDSD=34\&mp=1 (accessed on 10 October 2017).

33. National Development Council. Smart City Development under Land Planning. Public Gov. Quart. 2015, 3, $19-31$. Available online: https:/ / www.ndc.gov.tw/Advanced_Search.aspx?q=Public (accessed on 12 October 2017).

34. New Taipei City Yearbook 2017. Available online: http://www.bas.ntpc.gov.tw/content/?parent_id=10239 (accessed on 11 October 2017).

35. Taipei City Yearbook 2017. Available online: http:/ / dbas.gov.taipei / ct.asp?xItem $=61803 \& C t N o d e=6154 \&$ $\mathrm{mp}=120001$ (accessed on 20 October 2017).

36. Taoyuan City Yearbook 2017. Available online: http:/ / dbas.tycg.gov.tw/home.jsp?id=156\&parentpath=0\% 2C153\&mcustomize=picture_view.jsp\&dataserno=201403310001 (accessed on 20 October 2017).

37. Taichung City Yearbook 2017. Available online: http:/ /www.dbas.taichung.gov.tw /np.asp?ctNode=12288\& $\mathrm{mp}=113010$ (accessed on 13 October 2017).

38. Tainan City Yearbook 2017. Available online: http:/ / web.tainan.gov.tw / / account/list.asp?nsub=F3A300 (accessed on 18 October 2017).

39. Kaohsiung City Yearbook 2017. Available online: http://kcgdg.kcg.gov.tw/KCGSTAT/Page/ (accessed on 20 October 2017).

40. MOE 2017 Yearbook. Available online: https:/ / www.edu.tw /News_Content.aspx?n=829446EED325AD02\& sms=26FB481681F7B203\&s=B19AF3B0B4D7BFAC (accessed on 20 October 2017).

41. Yearbook of Environmental Protection Statistics 2017. Available online: https://www.epa.gov.tw/public/ Data/78239563771.pdf (accessed on 20 October 2017).

42. Taiwan Cultural Creative Industries Annual Report 2016. Available online: http://cci.culture.tw/cht/index. php? code=list\&ids=20 (accessed on 20 October 2017).

43. Zhang, Y.; Lu, H.; Luo, S.; Sun, Z.; Qu, W. Human-Scale Sustainability Assessment of Urban Intersections Based upon Multi-Source Big Data. Sustainability 2017, 9, 1148. [CrossRef]

(C) 2018 by the authors. Licensee MDPI, Basel, Switzerland. This article is an open access article distributed under the terms and conditions of the Creative Commons Attribution (CC BY) license (http:/ / creativecommons.org/licenses/by/4.0/). 